Relations industrielles

Industrial Relations

\title{
La déclaration de l'OIT relative aux droits fondamentaux au travail
}

Une nouvelle forme de régulation efficace?

\section{The ILO Declaration on Fundamental Principles and Rights at Work: A New and More Efficient Form of Regulation? La declaración de la OIT respecto a los derechos fundamentales del trabajo : una nueva forma de regulación eficaz}

Isabelle Duplessis

Volume 59, numéro 1, hiver 2004

Équité, efficience, éthique ? La régulation sociale de l'entreprise mondialisée

URI : https://id.erudit.org/iderudit/009127ar

DOI : https://doi.org/10.7202/009127ar

Aller au sommaire du numéro

Éditeur(s)

Département des relations industrielles de l'Université Laval

ISSN

0034-379X (imprimé)

1703-8138 (numérique)

Découvrir la revue

\section{Citer cet article}

Duplessis, I. (2004). La déclaration de l'OIT relative aux droits fondamentaux au travail : une nouvelle forme de régulation efficace ? Relations industrielles / Industrial Relations, 59(1), 52-72. https://doi.org/10.7202/009127ar
Résumé de l'article

Cet article explore l'efficacité de la Déclaration relative aux principes et droits fondamentaux au travail en tant que réponse aux défis posés par une mondialisation considérée essentiellement sur le plan économique. La Déclaration a été adoptée en 1998 par l'Organisation internationale du Travail (OIT) et visait à arrimer le développement économique au progrès social en établissant un corps universel de droits socio-économiques. Au regard des sources traditionnelles du droit international public, la Déclaration soulève pourtant un certain nombre de difficultés. Premièrement, elle s'apparente à un instrument de soft law, c'est-à-dire à un instrument incitatif dénué de force obligatoire. Deuxièmement, la Déclaration ne s'adresse pas directement aux acteurs réels de la mondialisation contemporaine, les entreprises mondialisées, mais aux États. À partir de ces critiques, la conjoncture ayant mené à l'adoption de la Déclaration fera l'objet d'une attention particulière et permettra de mieux mesurer les effets juridiques de cet instrument normatif au sein et à l'extérieur de l'OIT.
Tous droits réservés (C) Département des relations industrielles de l'Université Laval, 2004
Ce document est protégé par la loi sur le droit d'auteur. L'utilisation des services d'Érudit (y compris la reproduction) est assujettie à sa politique d'utilisation que vous pouvez consulter en ligne.

https://apropos.erudit.org/fr/usagers/politique-dutilisation/ 


\title{
La déclaration de l'OIT relative aux droits fondamentaux au travail Une nouvelle forme de régulation efficace?
}

\author{
ISABELLE DUPLESSIS
}

Cet article explore l'efficacité de la Déclaration relative aux principes et droits fondamentaux au travail en tant que réponse aux défis posés par une mondialisation considérée essentiellement sur le plan économique. La Déclaration a été adoptée en 1998 par l'Organisation internationale du Travail (OIT) et visait à arrimer le développement économique au progrès social en établissant un corps universel de droits socio-économiques. Au regard des sources traditionnelles du droit international public, la Déclaration soulève pourtant un certain nombre de difficultés. Premièrement, elle s'apparente à un instrument de soft law, c'est-à-dire à un instrument incitatif dénué de force obligatoire. Deuxièmement, la Déclaration ne s'adresse pas directement aux acteurs réels de la mondialisation contemporaine, les entreprises mondialisées, mais aux États. À partir de ces critiques, la conjoncture ayant mené à l'adoption de la Déclaration fera l'objet d'une attention particulière et permettra de mieux mesurer les effets juridiques de cet instrument normatif au sein et à l'extérieur de l'OIT.

La Déclaration relative aux principes et droits fondamentaux au travail ${ }^{1}$ [ci-après la Déclaration] a été adoptée le 18 juin 1998 par la Conférence internationale du Travail, organe plénier de l'Organisation internationale du Travail (OIT). Avec l'adoption de cette Déclaration, les États membres de

- Duplessis, I., Faculté de droit, Université de Montréal, Montréal, isabelle.duplessis@ umontreal.ca. L'auteure tient à remercier le professeur Gilles Trudeau pour ses conseils et ses encouragements.

1. Déclaration de l'OIT relative aux principes et droits fondamentaux au travail et son suivi, adoptée par la Conférence internationale du Travail à sa 86e session, Genève, 1998. 
l'OIT ont désormais l'obligation de respecter, promouvoir et réaliser, du seul fait de leur appartenance à l'Organisation et en l'absence de ratification des conventions pertinentes ${ }^{2}$, les principes concernant les droits fondamentaux suivant : la liberté d'association et la reconnaissance effective du droit de négociation collective ; l'élimination du travail forcé ou obligatoire, du travail des enfants, ainsi que de la discrimination en matière d'emploi et de profession.

Aux dires du Directeur général de l'OIT de l'époque, Michel Hansenne, l'adoption de la Déclaration se voulait une réponse aux défis soulevés par une mondialisation considérée jusqu'à maintenant seulement au plan économique, c'est-à-dire au travers d'une libéralisation du commerce international, de l'investissement et des flux de capitaux. Dès les années 1990, l'aspect inéquitable des retombées économiques de cette mondialisation d'un pays à l'autre, voire d'une région à l'autre, était pourtant devenu manifeste et intolérable pour certains observateurs de la scène internationale. Devant le rejet de l'idée d'une clause sociale par la toute nouvelle Organisation mondiale du commerce (OMC) et interpellée par sa propre mission institutionnelle, l'OIT devait agir rapidement en faveur de la justice sociale sous peine de subir une marginalisation progressive à l'intérieur du système international. C'est ce qu'elle fit en adoptant la Déclaration ${ }^{3}$. Ce document visait précisément à arrimer la croissance économique au progrès social en établissant un minimum de règles sociales universelles et applicables indépendamment des actes de volonté des États pris individuellement.

La Déclaration ne se limite pas à l'énonciation de principes et de droits au travail. Elle est en outre accompagnée d'un mécanisme de suivi, que l'on doit immédiatement distinguer d'un mécanisme de contrôle des obligations juridiques. Ce mécanisme est divisible en trois étapes. Premièrement, l'examen annuel est composé de rapports envoyés par les États dans lesquels ils décrivent les efforts consentis pour le respect des principes

2. Les huit conventions fondamentales pertinentes peuvent être regroupées en quatre catégories distinctes : la première catégorie regroupe la Convention $\left(n^{\circ} 29\right)$ sur le travail forcé, 1930, et la Convention ( $\left.n^{\circ} 105\right)$ sur l'abolition du travail forcé, 1957 ; la deuxième regroupe la Convention ( $n^{\circ}$ 87) sur la liberté syndicale et la protection du droit syndical, 1948, et la Convention ( $n^{\circ} 98$ ) sur le droit d'organisation et de négociation collective, 1949 ; la troisième, la Convention ( $n^{\circ} 100$ ) sur l'égalité de rémunération, 1951, et la Convention ( $\left.n^{\circ} 111\right)$ concernant la discrimination (emploi et profession), 1958 ; alors que la quatrième et dernière catégorie recoupe la Convention ( $\left.n^{\circ} 138\right)$ sur l'âge minimum, 1973, et la Convention ( $n^{\circ} 182$ ) sur les pires formes de travail des enfants, 1999.

3. Les débats ayant mené à l'adoption de la Déclaration en 1998 ont débuté en 1994. Au regard des négociations multilatérales internationales habituelles, ce laps de temps est relativement court. Pensons seulement aux travaux pour l'adoption de la Convention des Nations Unies sur le droit de la mer, adoptée à Montego Bay en 1982 qui se sont échelonnés sur un peu moins de dix ans. 
et droits fondamentaux de l'OIT énoncés dans les conventions fondamentales qu'ils n'ont pas encore ratifiées. Les organisations de travailleurs et d'employeurs représentatives reconnues ${ }^{4}$ peuvent aussi transmettre leurs commentaires respectifs sur la situation nationale décrite par leur gouvernement. Une introduction, rédigée par des experts indépendants, présente ensuite un résumé de tous les rapports et commentaires reçus et cherche à attirer l'attention du Conseil d'administration sur les aspects devant faire l'objet de discussions plus approfondies.

Dans un deuxième temps, un rapport global du Directeur général du Bureau international du travail (BIT) est soumis à la Conférence internationale du Travail. Comme son nom l'indique, le rapport en question entend donner une image de la situation mondiale au regard de l'une des catégories de principes et de droits fondamentaux chaque année. Toutes les catégories sont ainsi successivement examinées à l'intérieur d'un cycle de quatre ans. Ce rapport est élaboré à partir des données colligées par l'examen annuel concernant les conventions non ratifiées. Il s'appuie en outre sur toutes les autres sources officielles d'informations détenues par l'OIT, notamment celles contenues dans les rapports périodiques fournis par les États en vertu des conventions qu'ils ont ratifiées ${ }^{5}$. L'idée du rapport global est simple et s'enracine dans la constatation suivante : la ratification d'une convention par un État n'emporte pas nécessairement son respect dans les faits tout comme, à l'inverse, l'absence de ratification d'une convention ne signifie pas pour autant que les droits qui s'y rapportent ne sont pas respectés sur le territoire d'un État (Maupain 1999). Si l'examen annuel constitue une sorte d'auto-évaluation pour chaque État quant au respect des principes et droits énoncés dans les conventions qu'il n'a pas encore ratifiées, le rapport global intéresse au premier chef l'OIT elle-même en lui permettant de déterminer ses futures priorités en matière de coopération technique ; la coopération technique devant, par ailleurs, soutenir les efforts des États en vue de ratifier et mettre en ouvre les conventions fondamentales. Le rapport global concrétise ainsi l'obligation faite à l'Organisation d'aider ses Membres dans le respect des principes et droits fondamentaux en rationalisant l'allocation des ressources humaines et budgétaires de l'OIT ${ }^{6}$.

Le contenu du rapport global est discuté au sein de la Conférence internationale du Travail. Les résultats de cet échange sont ensuite acheminés

4. Se référer à l'article 23 qui lui-même renvoie à l'article 3 de la Constitution de l'Organisation internationale du Travail et Règlement de la Conférence internationale du Travail, Bureau international du travail (BIT), Genève, édition 1998.

5. Il s'agit du mécanisme de contrôle régulier prévu à l'article 22 de la Constitution de l'OIT.

6. Déclaration de l'OIT, paragr. 3. 
vers le Conseil d'administration qui, en collaboration avec le BIT, prépare le plan d'action, troisième et dernière étape du mécanisme de suivi de la Déclaration. Un plan est donc adopté par le Conseil d'administration pour chaque catégorie de principes et de droits à tour de rôle. Il définit les priorités de l'Organisation en matière de coopération technique pour la période quadriennale à venir. Il cherche aussi à encourager la levée de fonds et la générosité des donateurs afin de mieux poursuivre les activités promotionnelles de la Déclaration sur le terrain.

On peut évidemment s'interroger sur le bien-fondé de l'adoption de la Déclaration et de son suivi pour répondre aux défis de la mondialisation. On n'a d'ailleurs pas manqué de le faire. Le choix d'une telle forme de régulation pour l'établissement d'un programme aussi ambitieux que celui d'un socle social minimal universel est-il vraiment judicieux ? Les critiques formulées à l'encontre de la démarche de l'OIT sont nombreuses mais portent, pour l'essentiel, sur l'efficacité de la Déclaration à mettre en œuvre les droits fondamentaux au travail. Pour la plupart, elles dénoncent la forme de régulation retenue en tant que réponse inadéquate aux défis de la mondialisation. La Déclaration s'apparente en effet à un instrument de soft law, à une sorte de droit mou qui fragiliserait, selon certains juristes (Weil 1982), non seulement les droits qu'elle contient mais l'ensemble du système normatif international en étant dénué d'obligations juridiques précises à caractère obligatoire. Pour plusieurs commentateurs, un simple instrument à portée incitative ne saurait s'attaquer aux obstacles, bien réels ceux-là, rattachés au phénomène de la mondialisation économique. Avec la Déclaration, l'OIT aurait accouché ni plus ni moins d'une souris alors qu'elle avait le devoir d'aller plus loin que l'OMC, qui avait refusé en 1996 à Singapour de lier le commerce au développement social, en adoptant des obligations juridiques formelles concernant les droits fondamentaux des travailleurs.

Les critiques en ajoutent en considérant cette fois-ci les destinataires visés par la Déclaration. Conformément à la tradition du droit international classique, la Déclaration s'adresse aux États. Ce sont eux qui doivent modifier leurs comportements sur leur territoire et envers leur population afin de respecter les normes internationales auxquelles ils ont librement consenties au préalable. En conséquence, aux yeux de ses détracteurs, la Déclaration constitue tout au plus un coup d'épée dans l'eau. Elle ne s'adresse pas directement aux entreprises mondialisées, ces nouveaux acteurs de l'économie globale surpassant parfois en puissance les petites nations, mais encore une fois aux États.

Qu'en est-il véritablement ? Peut-on au contraire envisager la Déclaration de l'OIT comme une nouvelle forme de régulation efficace de l'économie globale ? Pour pouvoir juger de cette question, il faut d'abord prendre 
connaissance de la conjoncture ayant mené à privilégier l'adoption d'un tel instrument au lieu d'un autre au sein de l'OIT. Il faut aussi surtout souligner les objectifs poursuivis avec l'adoption de la Déclaration et les confronter aux résultats obtenus à ce jour. Après quelques remarques générales sur la Déclaration au regard des sources du droit international public, nous examinerons ses effets juridiques pour les États membres mais aussi pour l'Organisation elle-même. Notre propos s'achèvera sur la considération des effets de la Déclaration en dehors de l'OIT, c'est-à-dire sur tous les acteurs internationaux, notamment les entreprises mondialisées.

\section{LA DÉCLARATION ET LES SOURCES TRADITIONNELLES DU DROIT INTERNATIONAL PUBLIC}

Pour certains, le fait que la Déclaration soit un acte provenant d'une organisation internationale semble a priori jouer contre son efficacité. Cet acte peut en effet être rangé sous le sigle des résolutions ou recommandations adoptées par les organisations internationales à l'intention de leurs États membres. Il s'inscrit historiquement dans la pratique des « vœux », de ces souhaits exprimés par les organisations mais dont la réalisation repose sur la bonne volonté des États pris individuellement. Étant suspendue à l'acte volitif étatique, ce type d'instrument à portée incitative comporte un haut degré d'incertitude dans l'application de ses dispositions. Sa valeur juridique a pour cette raison fait l'objet de longs débats chez les juristes, à partir notamment de l'exemple des résolutions des Nations Unies (Sands et Klein 2001 ; Schermers et Blokker 1995). La preuve la plus éclatante du statut juridique indéterminé des actes unilatéraux des organisations internationales est qu'ils sont tout simplement absents de l'énumération de l'article 38 du Statut de la Cour internationale de Justice ${ }^{7}$ en tant que source de droit à laquelle la juridiction internationale peut se référer pour trancher les différends inter-étatiques.

La doctrine positiviste du droit international, qui a connu son apogée au XIX ${ }^{\mathrm{e}}$ siècle mais qui demeure néanmoins toujours importante pour saisir les fondements de la pensée juridique contemporaine, explique en grande partie les difficultés « ontologiques » des actes adoptés par les organisations

7. Cette énumération a été élaborée au début du siècle pour le fonctionnement de la Cour permanente de justice internationale et fait maintenant partie du Statut de la Cour internationale de Justice, annexé à la Charte des Nations Unies. Le moment de sa rédaction explique pour une part l'absence de mention des actes unilatéraux des organisations internationales en tant que source du droit international. Les organisations internationales en étaient encore au début du XX $\mathrm{X}^{\mathrm{e}}$ siècle à leurs premiers balbutiements. On ne pouvait vraisemblablement pas imaginer la multiplication et l'importance subséquente des organisations internationales dans le phénomène de réglementation internationale. 
internationales au regard des sources plus traditionnelles du droit que sont le traité ou la coutume. Suivant cette doctrine, les États sont les acteurs premiers sur la scène internationale en même temps que les créateurs exclusifs du droit international (Cutler 2001). Les obligations internationales ne peuvent découler que de la pratique des États et des accords passés entre eux. Elles se vérifient exclusivement de l'observation des faits et documents internationaux. La pensée jusnaturaliste, selon laquelle les obligations internationales sont déduites de principes d'inspiration divine ou des impératifs dictés par la Raison, est du même coup définitivement écartée avec le XIX ${ }^{\mathrm{e}}$ siècle (Jouannet 1998 ; Verdross 1927).

Dans cette structure juridique bien particulière, les organisations internationales sont des créatures dérivées des acteurs premiers et subordonnées à eux. À ce titre, elles ne peuvent prétendre imposer des obligations aux États sans leur consentement. Elles doivent se contenter de formuler des «vœux », des recommandations, dont la réalisation revient à chaque État individuellement. La valeur juridique des résolutions ou recommandations est finalement déterminée par les origines de l'organisation internationale : elle a été créée par les États dans un but de coopération internationale et ne doit à aucun moment se substituer à ses créateurs en agissant à l'image d'un parlement mondial. Les actes qu'elle peut adopter à l'égard de ses États membres sont incitatifs et ne relèvent pas de l'ordre du commandement du souverain comme dans la sphère du droit national. Or, la scène internationale n'a jamais été articulée autour d'une autorité centrale. L'organisation de son droit est donc complètement étrangère à la logique hiérarchique du commandement. De là à refuser aux résolutions ou recommandations des organisations internationales tout caractère contraignant, il n'y avait qu'un pas qui a vitement été franchi.

On constate aujourd'hui une augmentation sensible d'instruments normatifs à portée incitative sur le plan international (Abbott et al. 2000 ; Abbott et Snidal 2000 ; Deumier 2002). L'inflation du droit programmatoire - recommandations, principes directeurs, avis, standards, communiqués, etc. - semble accompagner l'expansion plus générale des organisations internationales dans tous les secteurs d'activités ayant une dimension internationale. N'en déplaise aux juristes positivistes, les instruments de soft law répondent à un besoin certain chez tous les acteurs internationaux confondus. À la lumière des circonstances, les États les préfèrent aux instruments juridiques traditionnels car ils sont plus faciles et plus rapides à négocier que les traités en bonne et due forme. On semble également les apprécier dans les domaines économique, technologique et environnemental, c'est-à-dire là où les obligations juridiques précises peuvent freiner dans le temps la fluidité des affaires commerciales ou les avancées scientifiques (Boustany et Halde 1997). 
Bref, dans les domaines comportant un facteur d'incertitude cognitive et de volatilité, les consignes vagues du type « les États prendront les mesures nécessaires » ou «les États feront des efforts » autorisent une adaptation rapide aux exigences fluctuantes de la réalité, tout en offrant une latitude aux États dans la mise en œuvre des normes. Cette souplesse des instruments de soft law ne signifie pas que les normes en question soient facultatives ou non obligatoires. Indépendamment de la largesse utilisée pour leur formulation et de la latitude autorisée pour leur mise en œuvre, les obligations internationales découlant des instruments de soft law comportent des effets juridiques et doivent être remplies de bonne foi par les États.

Au-delà des motifs d'ordre technique, des considérations politiques peuvent aussi empêcher les États de prendre des engagements juridiques formels sans pour autant effacer leur volonté d'adopter des standards normatifs. La nécessité de réglementer un champ d'activités internationales peut en effet commander une action normative même en l'absence d'un consensus sur l'adoption d'obligations juridiques formelles. On verra que la Déclaration de l'OIT de 1998 correspond à ce genre de situation. Dans un contexte paralysant de fracture idéologique entre les États, les organisations de travailleurs et celles des employeurs, l'OIT devait néanmoins agir rapidement devant les retombées inéquitables de la mondialisation économique et, plus largement, se repositionner à l'intérieur du système international de l'après Guerre froide. La Déclaration représentait à ce moment le maximum normatif à partir duquel un consensus au sein de l'Organisation pouvait encore être dégagé.

Un outil de soft law peut aussi parfois préparer les mentalités pour l'adoption ultérieure d'un instrument contraignant tel un traité. Il ne s'agit toutefois là que d'une hypothèse. La norme de soft law ne devrait pas être envisagée à tout coup comme l'antichambre de la règle de droit plus formelle. Nous soutenons plutôt que la Déclaration est un instrument de soft law possédant des effets juridiques en soi, immédiats, pouvant remplir les objectifs fixés par l'OIT au moment de son adoption et répondre ainsi au critère d'efficacité. La Déclaration n'est pas dénuée de tout caractère obligatoire et les États doivent veiller au respect des principes et droits qui y sont énoncés. En outre, son contenu se rattache bien souvent à de la hard law, c'est-à-dire à des obligations formelles et constitutionnelles des États membres de l'OIT.

La Déclaration est de surcroît chargée d'une symbolique puissante propre au $\mathrm{XX}^{\mathrm{e}}$ siècle. Elle est susceptible en conséquence de produire un supplément d'effets juridiques (Demers 1996). La Déclaration s'inscrit dans la tradition des grands documents internationaux consacrés aux droits de l'Homme. Elle a d'ailleurs été adoptée en 1998, année du cinquantième 
anniversaire de la Déclaration universelle des droits de l'Homme ${ }^{8}$, et incarne le premier instrument de cette envergure depuis la fin de la Guerre froide et le phénomène contemporain de libéralisation des échanges commerciaux. Le moment de son adoption est donc significatif et perpétue une philosophie d'inspiration humaniste dans une époque en pleine transformation. La Déclaration constitue un engagement renouvelé envers les mécanismes des droits de l'Homme et leur pertinence actuelle pour réglementer les conduites des États souverains sur leur propre territoire. Au plan socio-économique et sous réserve du développement de la pratique internationale, elle s'apparente à la Déclaration universelle des droits de l'Homme dont personne aujourd'hui ne conteste sérieusement la nature proprement juridique.

Signalons également l'emploi inusité de l'adjectif « fondamentaux » en matière de droits socio-économiques. Cette expression avait été jusqu'alors généralement réservée aux droits civils et politiques qui possédaient une prééminence, réelle ou souhaitée, dans la hiérarchie des droits de l'Homme. Les droits fondamentaux sont par définition applicables indépendamment de la situation nationale et des obstacles empiriques. Or, les droits socioéconomiques y figuraient difficilement puisque leur réalisation apparaissait conditionnelle aux ressources budgétaires de chaque pays. Cette logique conditionnelle semble avoir été abandonnée avec l'adoption de la Déclaration de 1998, du moins c'est la teneur du message que l'on peut légitimement interpréter.

Enfin, étant le produit d'un consensus quant aux droits fondamentaux au travail, la Déclaration comporte un potentiel d'efficacité à l'intérieur et à l'extérieur de l'OIT. Si ses dispositions sont incitatives, elles ne sont pas pour autant le fruit d'un tiraillement idéologique irrésolu entre les États membres. Cet aspect augmente les probabilités que la Déclaration soit respectée dans la pratique ${ }^{9}$. Il était aussi important pour la Conférence internationale du Travail d'introduire la Déclaration sous le couvert d'une consécration de droits préalablement et internationalement reconnus (Dufour 2003) et non comme une innovation. Pour reprendre textuellement le rapport de la Conférence ${ }^{10}$ :

« la déclaration ne vise pas en tant que telle à établir le caractère fondamental de certains droits. Leur prééminence résulte de l'objet sur lequel ils portent

8, Doc. N.U. A/810, p. 71 (1948).

9, L'importance du consensus international pour la production d'effets juridiques a déjà été soulignée par l'arbitre Dupuy à propos des résolutions de l'Assemblée générale des Nations Unies dans l'Affaire Texaco-Calasiatic, (1977) 104 J.D.I. 350.

10. Conférence internationale du Travail, Examen d'une éventuelle Déclaration de principes de l'Organisation internationale du Travail relative aux droits fondamentaux et de son mécanisme de suivi approprié, CIT, 86e session, 1998 (Rapport VII), p. 3-10. 
et du fait qu'ils ont déjà été reconnus comme fondamentaux à l'intérieur et à l'extérieur de l'OIT. En d'autres termes, les droits fondamentaux ne sont pas fondamentaux parce que la Déclaration le dit, mais la Déclaration le dit parce qu'ils le sont ».

\section{EFFETS JURIDIQUES DE LA DÉCLARATION À L'ÉGARD DES ÉTATS MEMBRES DE L'OIT}

Pour juger de l'efficacité de la Déclaration dans son objectif visant à arrimer le développement économique au progrès social, il faut savoir mesurer sur le terrain des États membres de l'OIT le respect des principes et des droits fondamentaux des travailleurs qu'elle consacre. Mais, au départ, comment expliquer le choix d'arrimer le développement économique au progrès social par l'intermédiaire du respect des droits fondamentaux des travailleurs plutôt que par l'idée d'une clause sociale ou encore un label social administré par l'OIT?

La question des droits fondamentaux des travailleurs et de l'adoption éventuelle d'une Déclaration a pris naissance au sein de l'OIT en 1994. Dans un rapport ${ }^{11}$ présenté à l'occasion du $75^{\circ}$ anniversaire de l'OIT à la Conférence internationale du Travail, le Directeur général examinait les valeurs constitutives de l'Organisation tout en cherchant le renforcement de ses moyens d'action dans un contexte de mondialisation économique. Somme toute, ce rapport s'assimilait à un procédé d'introspection nécessaire de la part de l'OIT afin de mieux se repositionner à l'intérieur du grand échiquier international. Cette introspection s'avérait d'autant plus incontournable, qu'en parallèle, des États industrialisés avaient milité pour une reconnaissance de certains droits des travailleurs lors de la conclusion du Cycle de l'Uruguay Round et de l'établissement d'un système commercial multilatéral. Le débat sur la clause sociale au sein des négociations menant à l'institution de la future $\mathrm{OMC}$, dont l'entrée en fonction sera le $1^{\text {er }}$ janvier 1995, ne s'était pas encore à ce moment soldé par un échec.

Quelques mois plus tard, toujours en 1995, au cours cette fois-ci du Sommet mondial des Nations Unies pour le développement social de Copenhague, un programme d'action est adopté. Ce programme se réfère explicitement à un noyau de droits fondamentaux des travailleurs et au mandat de l'OIT en la matière. Le Directeur général de l'OIT va en profiter pour lancer une vaste campagne invitant les États n'ayant pas ratifié toutes

11. Bureau international du travail, Des valeurs à défendre, des changements à entreprendre - La justice sociale dans une économie qui se mondialise : un projet pour l'OIT, rapport du Directeur général à la Conférence internationale du Travail, $81^{\mathrm{e}}$ session, Genève, 1994. 
les conventions portant sur les droits fondamentaux à le faire en précisant, dans l'affirmative, l'échéance prévue et, dans la négative, les obstacles de fond ou techniques les en empêchant. Ce n'est qu'à la $268^{\mathrm{e}}$ session du Conseil d'administration, soit en 1997, que l'idée d'une déclaration des droits fondamentaux des travailleurs est officiellement lancée par des représentants du groupe employeur. Les débats qui suivront dans l'année menant à son adoption seront néanmoins extrêmement houleux au sein de l'OIT.

Comment peut-on expliquer cette opposition à l'idée d'une déclaration alors qu'elle émane du groupe relativement conservateur des employeurs et qu'elle constitue tout au plus un instrument de soft law ? Une pareille force d'opposition se conçoit en effet beaucoup plus aisément si la proposition sous étude avait visé l'adoption d'un instrument juridique aux obligations précises et au mécanisme de contrôle rigoureux.

Il faut dire que la Déclaration fut victime de ses origines. Elle était en quelque sorte entachée par les débats sur la clause sociale. Un groupe d'États en développement s'était d'ailleurs battu avec succès contre l'inclusion d'une telle clause dans le cadre de l'OMC. Avec la fin des négociations de l'Uruguay Round en 1994, de fortes pressions avaient en effet été exercées pour établir un lien entre les sanctions commerciales et l'application des conventions fondamentales. Ce même groupe de pays craignait désormais la résurgence de la clause sociale au travers de l'OIT et l'utilisation de la Déclaration à des fins protectionnistes. De toute évidence, les milieux internationaux, quels qu'ils soient, n'étaient pas prêts à accueillir favorablement l'idée d'une clause sociale. Le paragraphe 5 de la Déclaration vient en quelque sorte conjurer les craintes en spécifiant que les normes du travail ne pourront servir à des fins commerciales protectionnistes ni dans l'objectif de réduire l'avantage comparatif d'un quelconque pays. L'inclusion de ce paragraphe interprétatif illustre a posteriori la force de l'opposition interne pendant les négociations de l'OIT menant à l'adoption de la Déclaration.

La crainte d'une clause sociale s'est traduite, notamment chez le groupe employeur, par un refus catégorique d'un instrument de hard law comportant des obligations juridiques supplémentaires pour les États membres ainsi qu'un mécanisme de contrôle affilié à ces obligations. L'OIT réussira à apaiser plusieurs inquiétudes en évoquant le caractère exclusivement déclaratif des droits fondamentaux mentionnés. Ces droits ne sont pas nouveaux et n'imposent aucune obligation juridique supplémentaire aux États membres puisque la liberté d'association, la protection des enfants et l'égalité de traitement et de chances se trouvaient déjà clairement affirmées dans la Constitution et dans la Déclaration de Philadelphie qui y est annexée. Le seul point où la Déclaration pouvait être interprétée comme une extrapolation, en l'absence de dispositions constitutionnelles explicites, est celui portant sur le travail forcé. Mais encore là, le bureau des services juridiques 
du BIT soutiendra avec brio que l'interdiction de ce type de pratique est inhérente aux principes constitutionnels de l'Organisation. Il était en effet raisonnable de déduire l'abolition du travail forcé de l'affirmation suivant laquelle le travail n'est pas une marchandise, ainsi que de celle garantissant aux travailleurs leur liberté d'association et leur égalité de chances ${ }^{12}$.

De manière plus compréhensive, l'OIT prétendra que l'ensemble des États membres se trouvent soumis, du seul fait de leur appartenance à l'Organisation et de leur adhésion à la Constitution, à un minimum d'obligations en matière de droits fondamentaux et ce, en l'absence même de ratification des conventions internationales pertinentes. Pour reprendre l'argumentation de l'Organisation, nous serions en présence d'une obligation constitutionnelle et non seulement d'une obligation conventionnelle quant au respect des droits fondamentaux des travailleurs. Parallèlement, l'OIT doit prendre tous les moyens possibles pour accomplir son mandat institutionnel, notamment en aidant ses États membres dans l'application universelle des droits fondamentaux des travailleurs. Les moyens en question peuvent consister à créer des nouveaux mécanismes de contrôle.

Cet argument des obligations constitutionnelles réciproques des États et de l'OIT pour convaincre tous les membres à adopter une déclaration des droits fondamentaux des travailleurs s'est toutefois avérée une arme à double tranchant. Il rappelait le fameux précédent de la création du Comité de la liberté syndicale de 1951. En se fondant sur l'affirmation de la liberté syndicale dans sa Constitution, l'OIT avait à cette époque mis sur pied une procédure spéciale de contrôle destinée à compléter les mécanismes réguliers du contrôle de l'application des normes internationales du travail. Cette procédure de plainte pour violation des droits syndicaux est ouverte tant aux gouvernements qu'aux organisations de travailleurs et d'employeurs contre les États, que ces derniers aient ou non ratifié les conventions pertinentes en matière de liberté syndicale (Gravel, Duplessis et Gernigon 2001). Au regard du droit international public, cette procédure est encore de nos jours hors du commun puisqu'elle ne nécessite ni la ratification des conventions pertinentes, ni le consentement exprès de l'État avant d'être mise en œuvre. L'argument constitutionnel revient donc mais cette fois pour légitimer l'adoption d'une déclaration des droits et d'un suivi. Au départ, l'Organisation évoquera, de manière analogique, la possibilité de créer une procédure similaire mais pour tous les droits fondamentaux et non seulement en matière de liberté syndicale ${ }^{13}$. Toutefois, faute de l'appui politique

12. Rapport VII, précité, note 10.

13. Bureau international du travail, L'action normative de l'OIT à l'heure de la mondialisation, rapport du Directeur général à la Conférence internationale du Travail, $85^{\mathrm{e}}$ session, Genève, 1997. 
général nécessaire, l'Organisation va vite écarter le précédent du Comité de la liberté syndicale de son argumentation visant à convaincre les États d'adopter la Déclaration. Elle distinguera ensuite soigneusement le suivi qui est rattaché à la Déclaration d'un mécanisme de contrôle proprement dit.

La conjoncture dans les années 1990 était tout simplement impropre à l'établissement d'un mécanisme de contrôle juridique pour des obligations découlant de conventions non ratifiées. Déjà, la création d'une telle procédure en 1951 avait posé de sérieuses difficultés pour certains États membres qui invoquaient l'inconstitutionnalité de ce mécanisme de contrôle, étant donné qu'il passait outre la ratification des conventions et violait de ce fait le principe de souveraineté étatique. Dans les années 1990, la conjoncture était encore défavorable aux initiatives juridiques de cet ordre. Ce refus d'une solution juridique peut d'ailleurs être retracé dans l'insistance à souligner le caractère strictement politique et promotionnel de la Déclaration. Le titre retenu est d'ailleurs symptomatique du phénomène. À aucun moment on ne parle de droits des travailleurs. On opère plutôt un glissement de sens du juridique au politique en référant « aux principes et aux droits fondamentaux au travail ». Cette expression gomme toute idée de conférer un recours à l'individu travailleur. Elle limite la Déclaration à ne reprendre que les éléments essentiels ou les objectifs largement définis par les conventions fondamentales ${ }^{14}$. Elle éloigne du même coup la possibilité d'utiliser les obligations détaillées des conventions fondamentales telles qu'interprétées par les différents mécanismes de contrôle, notamment par la Commission d'experts pour l'application des conventions et recommandations et par le Comité de la liberté syndicale. Cette précaution n'est pas négligeable si l'on rappelle seulement l'exemple du droit de grève déduit et longuement détaillé, au grand dam des employeurs, par les deux mécanismes de contrôle de l'OIT à partir des dispositions de la Convention ( $n^{\circ} 87$ ) sur la liberté syndicale et la protection du droit syndical. En insérant le mot « principes » avant «droits fondamentaux », on désamorce le référent juridique et on écarte plus aisément la jurisprudence élaborée par les organes de contrôle concernant les droits fondamentaux depuis les débuts de l'OIT en 1919.

L'OIT va venir à bout des résistances internes en soulignant le caractère promotionnel du suivi, échafaudé sur un mécanisme constitutionnel déjà existant. Pour ce faire, l'article 19(5)e) de la Constitution offrait le levier nécessaire pour l'établissement de l'examen annuel de la Déclaration. Cette disposition prévoit l'obligation pour l'État membre de faire rapport au Directeur général, à des périodes jugées appropriées par le Conseil

14. Voir les débats en plénière, notamment l'intervention de Monsieur Potter, délégué des employeurs, à la Conférence internationale du Travail, Rapport de la Commission de la Déclaration de principes, CIT, $86^{\mathrm{e}}$ session, 1998. 
d'administration, sur l'état de sa législation et de sa pratique concernant l'objet de la convention non ratifiée. L'État doit préciser « dans quelle mesure l'on a donné suite ou l'on se propose de donner suite à toute disposition de la convention par voie législative, par voie administrative, par voie de contrats collectifs ou par toute autre voie, et en exposant quelles difficultés empêchent ou retardent la ratification d'une telle convention ». Dans cette perspective, l'examen annuel, faisant partie du nouveau mécanisme de suivi de la Déclaration, n'est que l'usage renforcé et systématique de l'obligation préalablement prévue à l'article 19(5)e) de faire rapport à propos des conventions non ratifiées.

Les négociations ayant mené à l'adoption de la Déclaration exposent de manière éclatante un climat de méfiance généralisée envers le droit et les mécanismes juridiques pour arrimer le développement économique au progrès social. Ce climat avait été exacerbé par les débats internationaux de l'époque sur la clause sociale. Mais, l'OIT n'en demeurait pas moins confrontée aux conséquences sociales de la mondialisation économique et devait agir pour sa sauvegarde institutionnelle au sein du système juridique international. C'est pour cette raison qu'en dépit d'un climat défavorable à toute initiative juridique, il est aussi possible de déceler à cette époque une volonté commune d'agir chez les partenaires tripartites de l'Organisation. Parce qu'elle a été présentée justement sous la forme d'un instrument de soft law, la Déclaration a constitué la seule solution acceptable pour les partenaires.

En dépit des critiques à l'égard de ce type d'instrument normatif jugé trop mou et sans véritable force contraignante, la Déclaration a paradoxalement réussi à atteindre les objectifs pour lesquels elle a été officiellement adoptée. Elle visait explicitement à encourager la ratification des conventions fondamentales. Tous les programmes de coopération technique en témoignent et sont articulés autour de ce but. Or, depuis son adoption, une augmentation dans le nombre total des ratifications est incontestable ${ }^{15}$.

15. Pour la Convention ( $\left.n^{\circ} 29\right)$ sur le travail forcé, 1930, le nombre de ratification est passé de 137 en 1994, à 149 en 1998, à 162 en 2003. Pour la Convention ( $\left.n^{\circ} 105\right)$ sur l'abolition du travail forcé, 1957, le nombre est passé de 114 en 1994, à 135 en 1998, à 161 en 2003. Pour la Convention ( $n^{\circ}$ 87) sur la liberté syndicale et la protection du droit syndical, 1948, le nombre est passé de 112 en 1994, à 121 en 1998, à 142 en 2003. Pour la Convention ( $n^{\circ}$ 98) sur le droit d'organisation et de négociation collective, 1949, le nombre est passé de 126 en 1994, à 139 en 1998, à 153 en 2003. Pour la Convention ( $\left.n^{\circ} 100\right)$ sur l'égalité de rémunération, 1951, le nombre est passé de 126 en 1994, à 136 en 1998, à 161 en 2003. Pour la Convention ( $\left.n^{\circ} 111\right)$ concernant la discrimination (emploi et profession), 1958, le nombre est passé de 121 en 1994, à 131 en 1998, à 159 en 2003. Pour la Convention ( $\left.n^{\circ} 138\right)$ sur l'âge minimum, 1973, le nombre est passé de 46 en 1994, à 68 en 1998, à 130 en 2003. Pour la Convention ( $\left.n^{\circ} 182\right)$ sur les pires formes de travail des enfants, 1999, le nombre de ratification en 2003 était de 
Certes, il est difficile de se prononcer sur le lien de causalité et de soutenir avec certitude que la hausse des ratifications est directement attribuable à l'adoption de la Déclaration. Néanmoins, une chose est sûre : la coïncidence permet de croire qu'elle y a contribuée. L'ironie dans ce résultat est qu'avec la ratification des conventions fondamentales, tous les droits fondamentaux des travailleurs deviennent couverts par les mécanismes de contrôle de l'OIT. Alors que le choix de la Déclaration comme réponse à la mondialisation économique cherchait à éviter la création d'obligations juridiques supplémentaires et l'application des mécanismes de contrôle, voilà qu'on y arrive tout de même mais par des moyens politiques et promotionnels.

\section{EFFETS JURIDIQUES DE LA DÉCLARATION POUR L'OIT ELLE-MEMME}

Les débats en vue de l'adoption de la Déclaration ont démontré une prise de conscience au sein même de l'Organisation quant à la nécessité d'agir et d'agir rapidement pour contrer les conséquences inéquitables de la mondialisation contemporaine. Toutefois, cette prise de conscience n'allait pas jusqu'à embrasser sans discrimination toutes les mesures imaginables. Certains membres de l'Organisation refusaient nommément l'avenue juridique. L'OIT obtiendra finalement l'aval de ses mandants tripartites pour l'adoption de la Déclaration, un instrument normatif mais de soft law, c'est-à-dire se caractérisant pour sa souplesse et l'aspect promotionnel de son suivi. Plus spécifiquement, elle réussira à convaincre ses mandants premièrement en faisant valoir l'opportunité de la démarche constitutionnelle et, deuxièmement, en invoquant le caractère synallagmatique de la Déclaration pour les États membres et l'Organisation elle-même.

Pour mieux rallier le dernier groupe d'États opposé à l'idée de la Déclaration, l'OIT insistera sur la nécessité et l'opportunité d'agir. Son adoption a ainsi tour à tour pu être décrite ${ }^{16}$ comme « le troisième événement marquant de l'histoire de l'OIT » après la création de l'Organisation en 1919 et la Déclaration de Philadelphie ${ }^{17}$ rédigée suite à la grande dépression des

143 après seulement quelques années d'entrée en vigueur. Ces chiffres sont à jour en date du 17 août 2003, c'est-à-dire lors de notre dernière consultation du site officiel de l'OIT (http://webfusion.ilo.org/public/db/standards/normes/appl).

16. Les citations sont tirées des interventions de Monsieur Potter, délégué des employeurs, de Monsieur Willers, délégué gouvernemental de l'Allemagne, de Monsieur Blondel, délégué des travailleurs et de Monsieur Samet, délégué gouvernemental des États-Unis, débats en plénière, précités, note 14.

17. La Déclaration concernant les buts et objectifs de l'Organisation internationale du Travail a été adoptée par la Conférence internationale du Travail à sa $26^{\mathrm{e}}$ session le 10 mai 1944 à Philadelphie. Elle a ensuite été incorporée au texte de la Constitution en 1946. 
années trente et la fin de la Deuxième Guerre mondiale, comme un digne compromis dont "l'échec eût été dramatique », comme l'accomplissement de «quelque chose d'historique », comme « une victoire pour tous les Membres de l'OIT sans exception » confirmant le rôle spécifique de l'institution au sein des Nations Unies, et comme la réponse aux messages lancés par Copenhague mais surtout par la Réunion ministérielle de l'OMC tenue à Singapour en 1996.

Si ces affirmations faisaient partie de l'argumentaire plus général servi aux États membres afin de les persuader à adopter le document sous étude, elles informent tout de même sur la signification de la Déclaration pour l'Organisation elle-même. Son adoption représentait un repositionnement de l'OIT à l'intérieur du système international initié par des forces extérieures, plus particulièrement par l'OMC. Pour opérer ce repositionnement et justifier l'adoption de la Déclaration, l'OIT a utilisé de manière importante, comme elle a d'ailleurs pris l'habitude de le faire dans les moments de grandes tensions idéologiques, sa propre constitution afin de taire les divisions internes.

Tout d'abord, l'adoption de la Déclaration était considérée à l'époque par plusieurs observateurs intérieurs et extérieurs à l'OIT comme une priorité absolue dans l'agenda de l'Organisation afin de réaffirmer son mandat et surtout sa pertinence dans l'économie globale. Un besoin similaire s'était déjà fait sentir au cours de la Deuxième Guerre mondiale (Sohn 1999). Confrontée à l'échec et à la dissolution projetée de la Société des Nations, organisation internationale créée, rappelons-le, de concert avec l'OIT par le Traité de Versailles mettant un terme à la Première Guerre mondiale, l'OIT devait alors aussi réitérer de façon urgente sa mission et sa pertinence dans le monde de l'après-guerre. En réponse à cette situation, de hauts fonctionnaires du BIT avaient rédigé la Déclaration de Philadelphie qui réaffirmait essentiellement les buts et objectifs de l'Organisation. Ce faisant, ils lui ont évité la marginalisation au sein du système international émergent des Nations Unies, d'autant plus que le Conseil économique et social de la nouvelle organisation onusienne était responsable de la promotion des droits sociaux et économiques (Leary 1992) et pouvait, avec raison, être considérée comme un rival potentiel.

Dans cette perspective, l'OIT va interpréter le Sommet social de Copenhague de 1995, mais surtout les événements reliés à la Réunion ministérielle de l'OMC tenue à Singapour en 1996, comme des défis qu'on lui a lancés pour agir et réaffirmer la contemporanéité de sa mission institutionnelle en matière d'affaires sociales. À Copenhague, on s'était entendu sur l'existence d'un noyau de droits fondamentaux des travailleurs et sur la compétence de l'OIT pour le promouvoir. Que s'est-il produit à Singapour pour initier 
de manière décisive un mouvement à l'intérieur de l'OIT quant à la nécessité de se repositionner dans l'économie mondiale?

Rappelons que l'idée de la clause sociale, avancée notamment par les États-Unis, la France et la Norvège, avait été préalablement débattue au cours des pourparlers ayant mené à l'établissement de l'OMC et suscitait une opposition marquée de la part de plusieurs États africains, arabes et asiatiques. La résistance de ces pays à une quelconque discussion sociale pendant la Réunion de Singapour était à ce point prononcée qu'elle fut la cause d'un incident diplomatique mineur : l'invitation faite au Directeur général de l'OIT fut retirée pour mieux garantir aux États le caractère strictement commercial des futurs débats. Malgré cette précaution, la question sociale fut néanmoins abordée à la seule fin de dénoncer l'utilisation commerciale protectionniste des normes internationales du travail et reconnaître le mandat de l'OIT dans la protection et l'application des normes en question. De toute évidence, par cette Déclaration ministérielle, l'OMC refusait de lier la question sociale au commerce et semblait clore, du moins pour un temps, le débat sur l'opportunité d'une clause sociale dans son enceinte institutionnelle. L'OIT va choisir d'ignorer l'esprit du message de l'OMC, qui vise manifestement l'exclusion de la question sociale des affaires commerciales, pour retourner stratégiquement la Déclaration ministérielle de Singapour à son avantage en proposant une lecture littérale de celle-ci (Langille 1999). Le texte reconnaît en effet explicitement l'existence de droits fondamentaux des travailleurs et le mandat de l'OIT sur les questions sociales. L'OIT, en capitalisant sur la lettre du message de l'OMC, va réussir à surmonter ses propres divisions internes et convaincre l'ensemble de ses partenaires de la nécessité d'adopter la Déclaration relative aux principes et droits fondamentaux au travail.

Les tensions idéologiques entre les États industrialisés et les États en développement, déjà apparentes dans les débats sur la clause sociale au sein de l'OMC, ont aussi divisé l'OIT. En plus de faire appel aux messages extérieurs lancés par d'autres organisations internationales, l'OIT va se rabattre sur les valeurs axiologiques inscrites dans sa Constitution pour mieux persuader les États du bien-fondé de l'adoption de la Déclaration. Ce recours aux valeurs constitutionnelles de l'Organisation, pour dénouer les impasses idéologiques générées notamment par la Guerre froide, a été employé à maintes reprises dans l'histoire de l'OIT (Bonvin 1998). Il sert à dépassionner les débats et préparer le terrain aux compromis lorsque ni la négociation politique ni les mécanismes du droit positif n'y parviennent (Albert 2002 ; Teubner 2001). C'est en étant placées à distance des acteurs impliqués passionnellement dans un enjeu que les valeurs inscrites dans la Constitution de l'Organisation peuvent paradoxalement réunir les participants autour d'une solution. 
L'OIT va également justifier sa propre action en vue de faire adopter la Déclaration à la lumière de sa Constitution. Cet argument est important car toute la validité des actions entreprises par les organisations internationales repose sur l'interprétation de leur mandat constitutionnel (Amerasinghe 1996). L'OIT va donc s'appuyer sur l'obligation qui lui incombe, en vertu du préambule de sa Constitution et des articles II et III de la Déclaration de Philadelphie, de considérer tous les programmes d'action et les mesures d'ordre économique et financier susceptibles de promouvoir la réalisation de la justice sociale, et de seconder les États membres dans la mise en œuvre de ces programmes. Cette obligation de l'OIT est d'ailleurs spécifiquement reprise au paragraphe 3 de la Déclaration de 1998. Cette idée d'une obligation constitutionnelle de l'Organisation dans la réalisation de la justice sociale transforme en quelque sorte la Déclaration en un acte concerté conventionnel passé entre l'OIT et ses États membres. Le poids des obligations juridiques semble ainsi mieux réparti et non imposé unilatéralement par l'Organisation à ses États membres. La Déclaration est ainsi plus facile «à vendre » aux récalcitrants.

\section{EFFETS DE LA DÉCLARATION À L'EXTÉRIEUR DE L'OIT}

Si l'objectif réel de l'OIT avec l'adoption de la Déclaration était l'augmentation de la ratification des conventions fondamentales, cet objectif est actuellement atteint dans les faits. En ce sens, la Déclaration a été efficace et il importe peu de savoir tracer précisément le lien de causalité directe entre les deux événements que sont l'adoption de la Déclaration et la hausse des ratifications. Qu'est-il maintenant susceptible de se produire et comment la Déclaration peut-elle atteindre l'entreprise mondialisée alors qu'elle s'adresse principalement aux États, destinataires traditionnels de la règle de droit international public ? C'est toute la question des effets de la Déclaration en dehors de l'OIT qui est ici posée. Nous terminerons par une brève remarque qui se veut aussi une piste de réflexion pour l'avenir.

On a vu que l'adoption de la Déclaration avait, du point de vue de l'OIT, une finalité extérieure qui consistait essentiellement à repositionner l'Organisation au sein du système international, surtout face aux institutions économiques et financières telles que l'OMC, le FMI et la Banque mondiale. La Déclaration nous laisse l'impression d'être destinée, à l'instar des autres documents déclaratifs des droits de l'Homme, à des effets débordant le cadre strict de l'OIT. Elle contient une symbolique des droits de l'Homme qui transcende par définition les États pour bouleverser profondément toutes les mentalités, quelles qu'elles soient. Elle cherche à créer une opinion publique internationale avertie autour d'un certain nombre de droits socio-économiques pour qu'ensuite, le respect de la liberté syndicale et 
de la négociation collective, l'élimination de la discrimination en matière d'emploi et de profession et l'abolition du travail forcé et du travail des enfants apparaissent véritablement comme des impératifs catégoriques. À force d'incantation, la Déclaration est susceptible d'atteindre tous les acteurs internationaux, du moins au niveau des principes si ce n'est pas dans les droits détaillés par les différents organes de contrôle de l'OIT. Ses principes sont donc susceptibles d'être internalisés par les États mais aussi par les entreprises mondialisées.

Pour l'instant, la Déclaration a commencé subrepticement à coloniser le système économique dominant de nos jours toutes les autres disciplines (Frydman 2001). Si les institutions économiques et financières internationales n'ont pas d'obligations juridiques directes étant des tiers face à la Déclaration, elles doivent néanmoins, au minimum, s'abstenir de placer leurs membres en contradiction avec les engagements qu'ils auraient pu contracter auprès d'autres organisations internationales (Maupain 1999). Autrement dit, le caractère universel des droits fondamentaux au travail reconnus dans la Déclaration oblige indirectement les institutions économiques et financières internationales à veiller à leur respect au travers de leurs propres activités et programmes.

Un phénomène similaire se constate également dans l'adoption des codes de conduite des entreprises mondialisées et dans les labels sociaux développés sous l'impulsion d'initiatives privées. Toutes ces formes de régulation tendent à une relative uniformité quant à leur contenu. Les quatre catégories de droits fondamentaux au travail de l'OIT, notamment le droit très contesté à la liberté syndicale et à la négociation collective, constitueront bientôt une référence incontournable pour tous les acteurs internationaux, États ou entreprises mondialisées. Au-delà des débats sur sa nature juridique véritable et son contexte d'adoption, la Déclaration fait désormais partie du paysage international avec lequel il faudra composer, qu'on le veuille ou non.

\section{BIBLIOGRAPHIE}

Аввотт, K.W. et D. SNiDAL. 2000. « Hard and Soft Law in International Governance». International Organization, vol. 54, 421-456.

Aвbott, K.W., R.O. Keohane, A. MoravcsiK, A.-M. Slaughter et D. Snidal. 2000. «The Concept of Legalization». International Organization, vol. 54, 401-419.

Albert, M. 2002. «Governance and Democracy in European Systems : On Systems Theory and European Integration ». Review of International Studies, vol. 28, 293-309. 
AMERASINGHe, C.F. 1996. Principles of the Institutional Law of International Organizations, Cambridge : Cambridge University Press.

Bonvin, J.-M. 1998. L'organisation internationale du Travail. Étude sur une agence productrice de normes. Paris : Presses universitaires de France.

Boustany, K. et N. HALDE. 1997. « Mondialisation et mutations normatives : quelques réflexions en droit international ». Mondialisation des échanges et fonctions de l'État. F. Crépeau, dir. Bruxelles : Bruylant, 37-59.

CuTLER, A.C. 2001. «Critical Reflections ont the Westphalian Assumptions of International Law and Organization : A Crisis of Legitimacy ». Review of International Studies, 133-150.

DEMERS, V. 1996. Le contrôle des fumeurs : une étude de l'effectivité du droit. Montréal : Thémis.

Deumier, P. 2002. Le droit spontané. Paris : Économica.

Dufour, G. 2003. «La déclaration de l'OIT relative aux droits et principes fondamentaux du travail : nature et portée en droit international ». Mémoire de maîtrise inédit sous la direction de K. Boustany. Montréal : Département des sciences juridiques de l'Université du Québec à Montréal.

FRYDMAN, B. 2001. «Les nouveaux rapports entre droit et économie : trois hypothèses concurrentes ». Le droit dans la mondialisation. Une perspective critique. M. Chemillier-Gendreau et Y. Moulier-Boutang, dir. Paris : Presses universitaires de France, 59-76.

Gravel, E., I. Duplessis et B. Gernigon. 2001. Le Comité de la liberté syndicale : quel impact depuis sa création? Genève : Bureau international du travail.

JouAnNet, E. 1998. Emer de Vattel et l'émergence doctrinale du droit international classique. Paris : Pedone.

LANGILle, B.A. 1999. «The ILO and the New Economy : Recent Developments ». The International Journal of Comparative Labour Law and Industrial Relations, vol. 15, 229-257.

LEARY, V.A. 1992. «Lessons from the Experience of the International Labour Organisation». The United Nations and Human Rights : A Critical Appraisal. P. Alston, ed. Oxford : Clarendon Press, 580-619.

MAupAIn, F. 1999. «L'OIT, la justice sociale et la mondialisation». Recueil des cours de l'Académie internationale de droit international, vol. 278, 201-396.

SANDS, P. et P. KLEIN. 2001. Bowett's Law of International Institutions. $5^{\mathrm{e}}$ édition. London : Sweet \& Maxwell.

SCHERMERS, G. et N. BlOKKER. 1995. International Institutional Law. $3^{\mathrm{e}}$ édition révisée. The Hague : Martinus Nijhoff Publishers.

SoHN, L.B. 1999. « The Contribution of the International Labor Organization to the Development of the Concept of Economic, Social and Cultural Rights ». Mélanges en l'honneur de Nicolas Valticos : Droit et justice. Paris : Pedone, 595-606.

Teubner, G. 2001. «Un droit spontané dans la société mondiale». Le droit saisi par la mondialisation. C.-A. Morand, dir. Bruxelles : Bruylant, 197-220. 
VERDROSS, A. 1927. «Le fondement du droit international». Recueil des cours de l'Académie de droit international, vol. 16, 251-321.

WEIL, P. 1982. «Vers une normativité relative en droit international?». Revue de droit international public, vol. 86, 5-47.

\section{SUMMARY}

\section{The ILO Declaration on Fundamental Principles and Rights at Work: A New and More Efficient Form of Regulation?}

This article examines the efficiency of the ILO Declaration on Fundamental Principles and Rights at Work and its Follow-up as a response to the challenges brought forth by a globalization which, until now, has been mainly understood in economic terms. The International Labour Organization adopted in June 1998 the ILO Declaration to ensure that social progress goes hand in hand with economic growth. In order to maintain the link between the two, the ILO Declaration sets forth the obligation to all members, even if they have not ratified the Conventions recognized as fundamental both outside and inside the ILO Organization, to respect, promote and to implement the principles which are the subject of those Conventions. These include: the freedom of association and the effective recognition of the right to bargain collectively; the elimination of all forms of forced labour; the effective abolition of child labour; and the elimination of discrimination in respect of employment and occupation. In accordance with the Constitution, this obligation to all Members arises from the very fact of membership in the Organization. Thus the Declaration seeks to ensure a minimal universal social basis to certain international rules, independently of differences between States.

However, the Declaration presents certain problems with regard to the traditional sources of public international law. First, it resembles a soft law instrument, meaning an instrument that may influence and encourage but which has no obligatory force. Furthermore, this instrument stems from an international organization, which is a secondary subject of public international law in comparison with the sovereign State. Second, the Declaration does not directly address itself to the actors of contemporary globalization such as multinational firms and companies, but also the States. In this respect, how could such an instrument really tackle the inequitable effects of contemporary economic globalization? Did the 1998 ILO declaration give birth to a mouse?

In the light of these criticisms, this article describes the circumstances of the negotiations which led to the adoption of the Declaration. The specific 
context of the time shows how there was a climate of internal ideological tension between the ILO Members regarding what kind of strategy to follow. There was also an institutional necessity for the Organization to act in order to reposition itself on the international chessboard at the end of the $\mathrm{XX}^{\text {th }}$ century. The adoption of the Declaration constituted the smallest common denominator between the Members. In order to convince its Members, the ILO made use of its constitutional resources - as it has done so in the past.

Finally, the legal effects of the Declaration, inside as well as outside the ILO, are examined. Ironically, even though the ILO Declaration is a soft law instrument and was presented to Members as such, it is able to achieve the same results as a hard law instrument would have done in terms of the ratification of fundamental conventions. Moreover, in thinking about the impact beyond the ILO, in setting minimal social standards for all, multinational firms and alike, the ILO Declaration is building a strong public opinion around those rights at work. This, in turn, is surely a way to implement them efficiently. 